\title{
Unique electron polarimeter analyzing power comparison and precision spin-based energy measurement
}

\author{
J. M. Grames, ${ }^{*}$ C. K. Sinclair, ${ }^{\dagger}$ J. Mitchell ${ }^{\ddagger}$ E. Chudakov, H. Fenker, A. Freyberger, D.W. Higinbotham, M. Poelker, \\ M. Steigerwald, ${ }^{\S}$ and M. Tiefenback \\ Thomas Jefferson National Accelerator Facility, Newport News, Virginia 23606, USA \\ C. Cavata, S. Escoffier, F. Marie, T. Pussieux, and P. Vernin \\ CEA Saclay, DSM/DAPNIA/SPHN, 91191 Gif sur Yvette Cedex, France \\ S. Danagoulian \\ North Carolina Agricultural and Technical State University, Greensboro, North Carolina 27411, USA \\ V. Dharmawardane \\ Old Dominion University, Norfolk, Virginia 23529, USA \\ R. Fatemi, K. Joo, and M. Zeier \\ University of Virginia, Charlottesville, Virginia 22904-4714, USA \\ V. Gorbenko \\ Kharkov Institute of Physics and Technology, Kharkov 61108, Ukraine
}

R. Nasseripour and B. Raue

Florida International University, Miami, Florida 33199, USA

R. Suleiman

Massachusetts Institute of Technology, Cambridge, Massachusetts 02139-4307, USA

B. Zihlmann

Vrije Universiteit, 1081 HV, Amsterdam, The Netherlands

(Received 17 March 2004; published 19 April 2004)

\begin{abstract}
Precision measurements of the relative analyzing powers of five electron beam polarimeters, based on Compton, Møller, and Mott scattering, have been performed using the CEBAF accelerator at the Thomas Jefferson National Accelerator Facility (Jefferson Laboratory). A Wien filter in the $100 \mathrm{keV}$ beam line of the injector was used to vary the electron spin orientation exiting the injector. High statistical precision measurements of the scattering asymmetry as a function of the spin orientation were made with each polarimeter. Since each polarimeter receives beam with the same magnitude of polarization, these asymmetry measurements permit a high statistical precision comparison of the relative analyzing powers of the five polarimeters. This is the first time a precise comparison of the analyzing powers of Compton, Møller, and Mott scattering polarimeters has been made. Statistically significant disagreements among the values of the beam polarization calculated from the asymmetry measurements made with each polarimeter reveal either errors in the values of the analyzing power or failure to correctly include all systematic effects. The measurements reported here represent a first step toward understanding the systematic effects of these electron polarimeters. Such studies are necessary to realize high absolute accuracy (ca. 1\%) electron polarization measurements, as required for some parity violation measurements planned at Jefferson Laboratory. Finally, a comparison of the value of the spin orientation exiting the injector that provides maximum longitudinal polarization in each experimental hall leads to an independent and very precise (better than $10^{-4}$ ) absolute measurement of the final electron beam energy.

DOI: 10.1103/PhysRevSTAB.7.042802

PACS numbers: 29.25.Bx, 29.27.Hj, 29.30.Dn, 13.60.Fz
\end{abstract}

*Corresponding author.Electronic address: grames@jlab.org

${ }^{\dagger}$ Present address: Wilson Laboratory, Cornell University, Ithaca, NY 14853, USA.

${ }^{\ddagger}$ Present address: Renaissance Technologies Corporation, 600 Route 25A, East Setauket, NY 11733-2841, USA.

${ }^{\S}$ Present address: Carl Zeiss Lithos GmbH, Carl Zeiss Straße 50, D-73447 Oberkochen, Germany.

\section{INTRODUCTION}

The use of beams of polarized electrons in nuclear and high energy physics experiments provides an important degree of freedom for understanding fundamental interactions. For example, the spin structure of nucleons can be explored using a beam of longitudinally polarized 
electrons in conjunction with either a polarized target or recoil polarimetry or by studying parity violation in the scattering of longitudinally polarized electrons from unpolarized targets. In general, the uncertainty in the knowledge of the electron beam polarization is a significant contribution to the overall error bar in these measurements. While experiments using polarized targets or recoil polarimetry do not generally require the highest precision electron polarimetry, this is not the case with parity violation measurements. For some planned parity violation measurements, absolute knowledge of the electron beam polarization at the $1 \%$ level is desired. This is beyond the present state of the art in electron polarimetry.

All electron beam polarimeters developed to date for electron energies above a few $\mathrm{keV}$ rely on the spin dependence in one of three electron scattering processes: Mott, Compton, or Møller scattering. The spin dependence of each of these three scattering processes gives the physics analyzing power. The scattering target may be polarized, as in Compton or Møller polarimeters, or unpolarized, as in Mott polarimeters. In the former case, the analyzing power is the product of the physics analyzing power and the target polarization, and in the latter case, it is simply the physics analyzing power. The effective analyzing power of a polarimeter incorporates many additional details, such as detector acceptance and resolution, systematic effects, and backgrounds. In each case, the spin dependent cross section yields a measurable asymmetry in the scattered flux equal to the product of the beam polarization and the effective analyzing power. In general, no polarimeter measures the total beam polarization. Rather, the particular components of the beam polarization measured are determined by the polarimeter design.

The most desirable characteristic of any polarimeter is a large and well-known effective analyzing power. However, precise knowledge of the effective analyzing power is limited because it is not generally a directly measured quantity. Direct measurement of the effective analyzing power of a particular scattering process requires very difficult double scattering experiments. Small effective analyzing powers, low cross sections, and discrimination against backgrounds make such experiments highly impractical in most cases. For all the polarimeters used in the measurements reported here, and indeed in almost all cases, the effective analyzing power of a polarimeter is determined by computer simulation. These simulations include not only the physics asymmetry of the underlying scattering process, but also the details of the real detector, systematic effects to the extent they are identified, multiple scattering, and background effects. It is quite possible that the true value of the effective analyzing power of a polarimeter differs from the simulated value by more than the uncertainty as determined by simulation. The importance of the Levchuk effect $[1,2]$ to the effective analyzing power of
Møller polarimeters, which emerged long after Møller polarimeters became a common way of measuring electron beam polarization, is a good example of this reality.

The presence of five polarimeters at Jefferson Laboratory, using all three basic electron scattering interactions, led to a joint effort by all the polarimeter groups at the laboratory to precisely compare the effective analyzing powers of these polarimeters, by using each to measure the polarization of the same beam. The effective analyzing power for each polarimeter was determined through simulations done by the experimental groups that constructed and commissioned these polarimeters. The measurements reported here have the aim of revealing any errors in the effective analyzing powers of the five polarimeters.

\section{Experimental plan and CEBAF accelerator}

The experimental plans to compare the effective analyzing powers of Compton, Møller, and Mott polarimeters, using the CEBAF accelerator, are discussed in the remainder of this section. The CEBAF accelerator [3] shown in Fig. 1 is a $6 \mathrm{GeV}, 200 \mu \mathrm{A}$ continuous beam electron accelerator in which a beam of highly polarized electrons is recirculated up to 5 times through two superconducting linear accelerators (linacs). Radio frequency (rf) separators allow beams to be extracted after any recirculation pass and delivered simultaneously to three experimental halls, shown as A, B, and C in Fig. 1. For this experiment, a five-pass beam was delivered to all three experimental halls.

The locations of the five polarimeters are indicated in Fig. 1, and their operating parameters are given in Table I. All five polarimeters are discussed in greater detail in the following sections.

The accelerator delivers a highly polarized beam from the injector simultaneously to all experimental hall polarimeters. This offers the advantage that all polarimeters receive the same magnitude of beam polarization. However, since the total precession from the injector to each experimental hall is different, the measurable components of the beam polarization are generally not equal at the various polarimeters.

Although the nine recirculation arcs and the experimental hall beam transport lines lie in different horizontal planes, there is, with very high precision, no net vertical bend between the injector (and its Mott polarimeter) beam line and the beam lines through the various experimental hall polarimeters. As a result, any vertical component of polarization at the exit of the injector appears unaltered at the experimental hall polarimeters (for further explanation, see Sec. IIIB). In the horizontal plane, the beam polarization undergoes a large precession from the injector to the various experimental hall polarimeters, due to the large net horizontal bend angle and the high beam energy. Thus, in general, the polarization at 


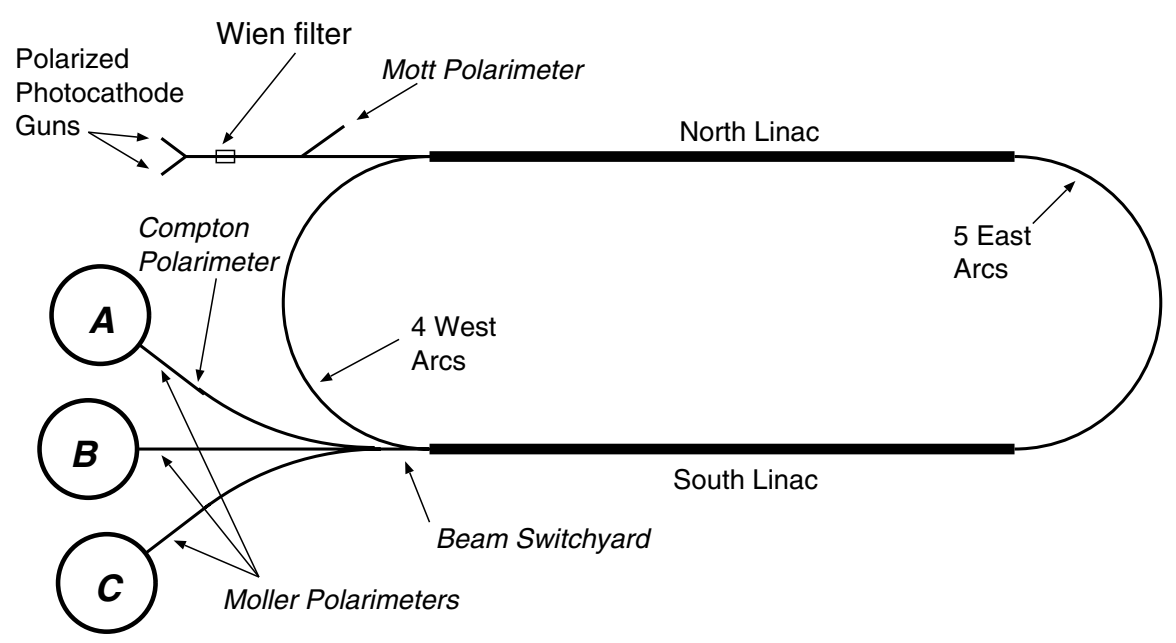

FIG. 1. Schematic of the CEBAF accelerator showing elements of the experiment.

TABLE I. Operating parameters of the five Jefferson Laboratory electron polarimeters.

\begin{tabular}{lcccc}
\hline \hline Polarimeter & Reaction & Intensity & Target & Measurement \\
\hline Injector Mott & $\vec{e}+Z A$ & $5 \mu \mathrm{A}$ & Gold $(1 \mu \mathrm{m})$ & $P_{x}, P_{y}$ \\
Hall A Compton & $\vec{e}+\vec{\gamma}$ & $100 \mu \mathrm{A}$ & Photon $(\lambda=1064 \mathrm{~nm})$ & $P_{z}$ \\
Hall A Møller & $\vec{e}+\vec{e}$ & $500 \mathrm{nA}$ & Supermendur $(13 \mu \mathrm{m})$ & $P_{z}, P_{x}$ \\
Hall B Møller & $\vec{e}+\vec{e}$ & $5 \mathrm{nA}$ & Permendur $(25 \mu \mathrm{m})$ & $P_{z}, P_{y}$ \\
Hall C Møller & $\vec{e}+\vec{e}$ & $2 \mu \mathrm{A}$ & Iron $(4 \mu \mathrm{m})$ & $P_{z}$ \\
\hline \hline
\end{tabular}

each experimental hall polarimeter has a longitudinal component parallel to the beam momentum, a horizontal component transverse to the beam momentum (hereafter referred to as "horizontal"), and a small vertical component. Clearly in those cases where the longitudinal component is small, the horizontal component is large. Since all four experimental hall polarimeters have been designed to measure the longitudinal polarization, they may be subject to significant systematic effects arising from the large horizontal component when the longitudinal component is small, as in some of the measurements reported here.

The uncertainty in the total precession between the injector and the experimental hall polarimeters complicates precise measurements of the relative analyzing powers. The solution to this problem is to conduct the measurements in a way that does not rely on only one measurement of a single component of the beam polarization. This is accomplished by adjusting the orientation of the beam polarization with a spin rotator, in this case a Wien filter, common to all beams. The beam polarization measured by each polarimeter can then be plotted against the common spin orientation. A fit to these data yields both the magnitude of the measured polarization and the spin orientation at the injector that results in the maximum value of the measured polarization at each polarimeter. The importance of longitudinally polarized electron beams, stated earlier, means that the experimental hall polarimeters have all been constructed to measure the longitudinal component of the beam polarization, whereas the injector Mott polarimeter can measure only the transverse component of the beam polarization.

\section{ELECTRON POLARIMETERS AT JEFFERSON LAB}

The five electron polarimeters are described in the sections below. The references given in each section provide additional details.

\section{A. Mott polarimeter in the injector}

To support a reliable, high precision measurement of the beam polarization in the electron injector a $5 \mathrm{MeV}$ Mott scattering polarimeter (see Fig. 2) has been developed [4,5]. The Mott scattering asymmetry arises from the spin-orbit coupling between the electron spin and its orbital angular momentum in the Coulomb potential of the target nucleus. The polarimeter is located in the $5 \mathrm{MeV}$ region of the injector on a dedicated beam line $12.5^{\circ}$ from the injector beam line. The polarimeter measures both transverse components of the beam polarization and has been studied [6] over a range of energies $(2-8 \mathrm{MeV})$ with target foils of varying thickness and three atomic numbers (79 for gold, 47 for silver, and 29 for copper). The 


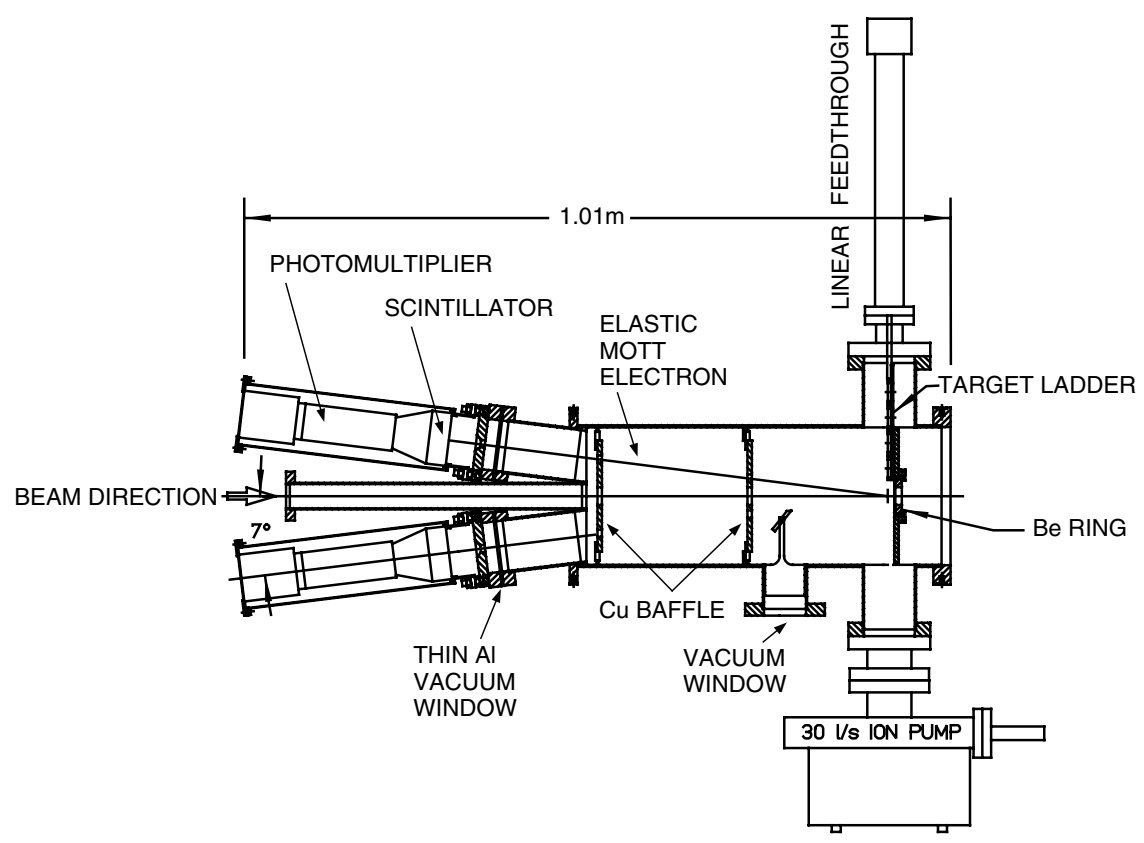

FIG. 2. Schematic of the injector Mott polarimeter as installed in the $5 \mathrm{MeV}$ region of the CEBAF injector.

maximum analyzing power occurs at an electron scattering angle close to $172^{\circ}$ for all values of the beam energy and atomic number measured.

The polarimeter has four electron detector arms, two in the horizontal plane and two in the vertical plane, with each member of a pair separated by $180^{\circ}$ in azimuth. Internal collimation defines the acceptance of the individual detector arms and assures that each detector views only the central area of the target foil. A combination of thin and thick scintillation counters are used in coincidence to discriminate against photons. The energy of the elastically scattered $5 \mathrm{MeV}$ electron is totally absorbed in the thick detector, and the signal from this detector is required to be above a suitable threshold. The rf time structure on the electron beam allows the use of timeof-flight detection, which discriminates against electrons scattered from material other than the primary target.

The analyzing power of the standard $1 \mu \mathrm{m}$ gold target foil used in these measurements was determined by measuring the scattering asymmetry for a large number of gold foils with thicknesses ranging between $50 \mathrm{~nm}$ and $5 \mu \mathrm{m}$. These measurements allowed a high precision extrapolation to zero foil thickness. The analyzing power of a zero thickness foil is taken to be that calculated for scattering by a single atom. At the $5 \mathrm{MeV}$ beam energy used in the experiment, the effective analyzing power, or effective Sherman function, for the standard foil is $S_{\text {eff }}=$ $-0.4008 \pm 0.0014 \pm 0.0040$. The first uncertainty is instrumental and the second is the theoretical uncertainty in the calculated Sherman function for single atom scattering. The theoretical uncertainty includes the uncertainties in the radiative correction and the nuclear size effect. As the target is unpolarized, asymmetries arising from any longitudinal polarization component would be parity violating, and are thus negligible.

\section{B. Compton polarimeter in experimental Hall A}

The first of the two electron polarimeters in the Hall A beam line is a Compton polarimeter [7-9] (see Fig. 3). The Compton scattering asymmetry results from the interaction of a longitudinally polarized electron beam and a circularly polarized photon (target) beam. The photon beam circulates in a Fabry-Perot cavity centered within a chicane comprised of four vertical bend dipole magnets. An external laser (250 $\mathrm{mW}$ at $1064 \mathrm{~nm})$ is locked to and fills the cavity with circularly polarized light $\left(P_{\text {circ }}>99 \%\right)$. The gain of the cavity results in about $1200 \mathrm{~W}$ of circulating optical beam power. A remotely controlled quarter-wave plate external to the Fabry-Perot cavity may be rotated to select either state of circular polarization and is used to reverse the overall sign of the scattering asymmetry for systematic correction. The optical transport mirrors are oriented to maintain the optical polarization into the cavity. The cavity maintains the photon beam at a small angle to the incident electron beam. Compton backscattered photons exit through the third chicane dipole and are detected using a $\mathrm{PbWO}_{4}$ crystal calorimeter. A detector for the scattered electron also exists, but was not used in this experiment. The effective Compton analyzing power depends on the beam energy and is a modeled number, rather than one calculated from first principles. The effective analyzing power is modeled for each run (electron spin orientation) separately. For this experiment the effective analyzing 


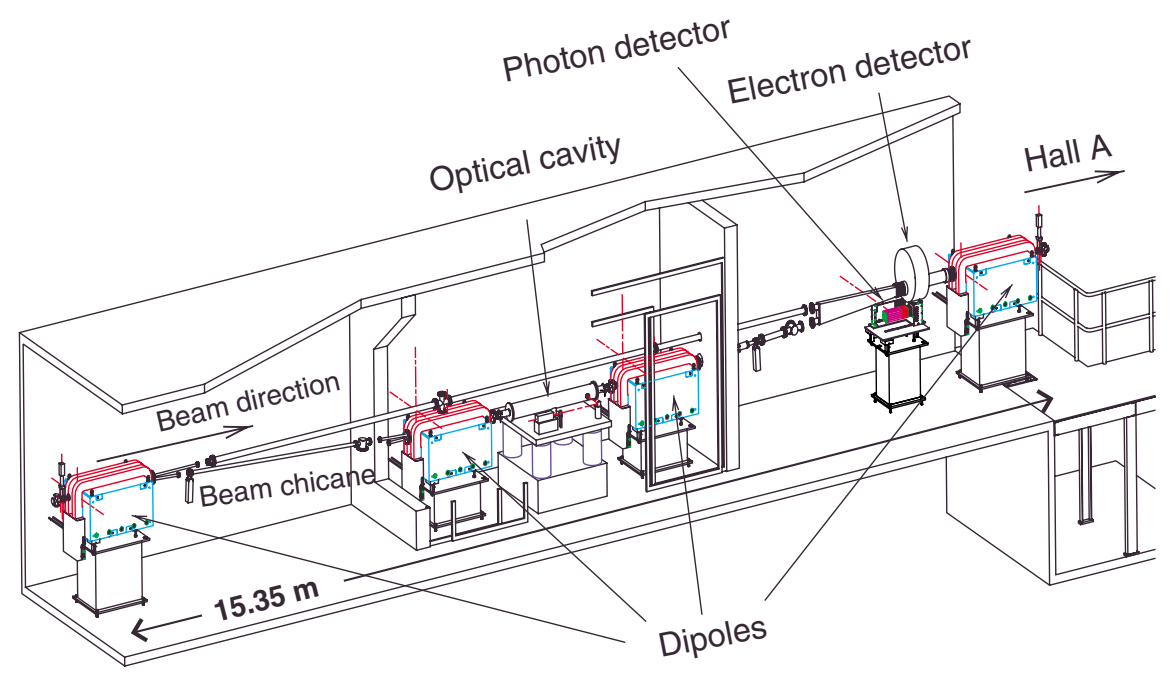

FIG. 3. (Color) Schematic of the Hall A Compton polarimeter.

power is $A_{\text {eff }}=0.024$. The systematic uncertainty ranged between $0.6 \%$ and $2.6 \%$ over the range of spin angles of this experiment. The systematic uncertainty of the laser polarization is $1 \%$.

\section{Møller polarimeter in experimental Hall A}

Downstream of the Compton polarimeter is a Møller polarimeter [10] (see Fig. 4) consisting of a solid polarized target, a magnetic spectrometer (three quadrupoles and one dipole), and lead glass and scintillator detectors. The polarimeter uses either of two iron-alloy targets (supermendur) which are polarized by a weak $240 \mathrm{G}$ longitudinal magnetic field created by a pair of Helmholtz coils. The target polarization is measured to be $0.0795 \pm 0.0024$. The Møller pairs (scattered incident electron and recoil target electron), centered about $90^{\circ}$ in the center of mass, are detected in coincidence. The targets are $13 \mu \mathrm{m}$ thick and are positioned at angles of $\pm 20^{\circ}$ horizontally with respect to the beam, giving an effective target thickness of $38 \mu \mathrm{m}$. The longitudinal component of the beam polarization is determined by using the oppositely oriented foils to subtract the asymmetry arising from the horizontal component of the beam polarization. The targets are cooled by conduction through the target support. At $500 \mathrm{nA}$ beam current, the estimated target temperature rise is several degrees Kelvin and the associated relative change in target polarization is estimated to be below $0.1 \%$. The simulated value of the physics analyzing power of the polarimeter is $A_{z z}=-0.7600 \pm 0.0023$. The effective analyzing power is $A_{\text {eff }}=-0.0604 \pm 0.0018$.
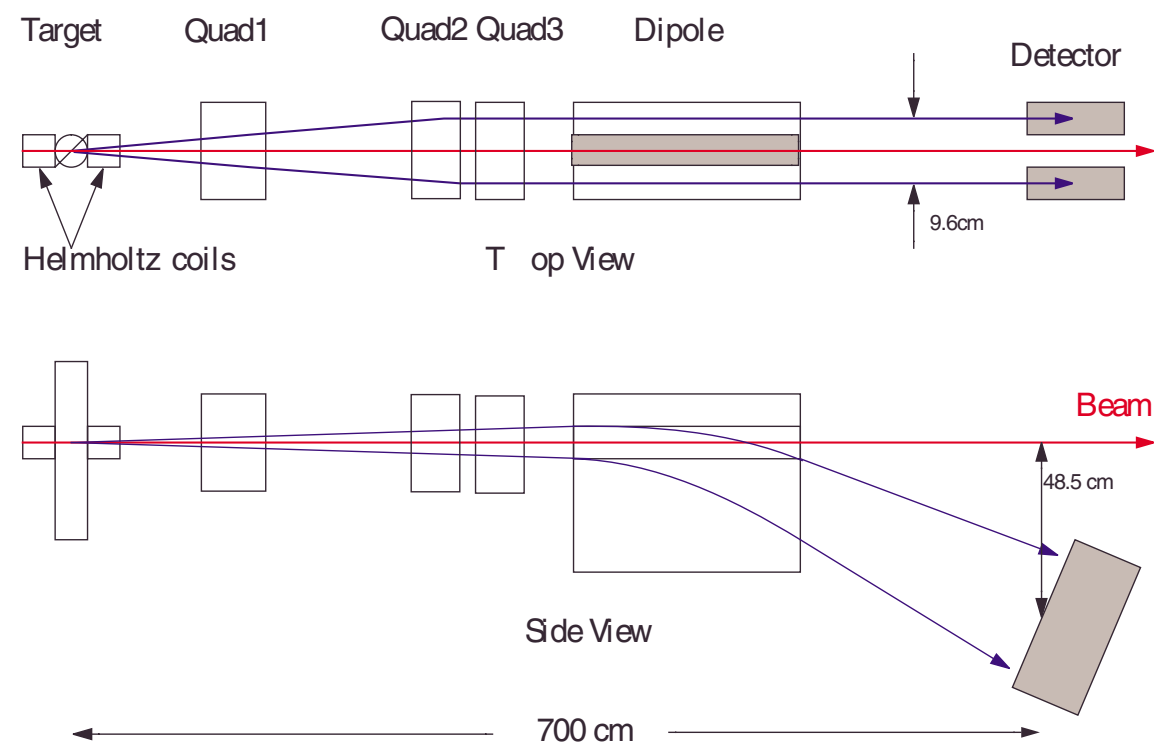

FIG. 4. (Color) Schematic of the Hall A Møller polarimeter. 


\section{Møller polarimeter in experimental Hall B}

The Hall B Møller polarimeter [11] uses a polarized target similar to that in Hall A, a two quadrupole magnetic spectrometer, and coincidence detection of the scattered and recoil electrons. Two $25 \mu \mathrm{m}$ thick permendur foils $(49 \% \mathrm{Fe}, 49 \% \mathrm{Co}, 2 \% \mathrm{~V})$ are oriented with their planes at $\pm 20^{\circ}$ vertically with respect to the beam direction, giving an effective target thickness of $73 \mu \mathrm{m}$. The target is polarized to $\simeq 7.5 \%$ along the beam direction by the $120 \mathrm{G}$ longitudinal field of a pair of Helmholtz coils. This polarimeter is operated at low beam currents (a few nanoamps average) typical of Hall B experiments. In contrast to the Hall A Møller polarimeter the longitudinal component of the beam polarization is determined by subtracting the asymmetry arising from the vertical component of the beam polarization. However, only one target was used for this experiment, and no correction for the vertical component was made. The physics analyzing power is simulated to be $A_{z z}=-0.7826 \pm 0.0062$. The effective analyzing power is $A_{\text {eff }}=-0.0587$.

\section{E. Møller polarimeter in experimental Hall C}

The Hall C Møller polarimeter [12,13] (see Fig. 5) consists of a polarized iron target, a two quadrupole magnetic spectrometer, a collimator system, and lead glass and scintillation detectors. The target is a pure iron foil positioned normal to the incident beam within a $3 \mathrm{~T}$ longitudinal magnetic field created by a pair of superconducting Helmholtz coils. In this target design the out-of-plane magnetization is saturated in the external field, yielding a target polarization $P_{z}=0.0800 \pm$ 0.0004 . The uncertainty in the target polarization includes an estimate of the effect of target heating by a $2.5 \mu \mathrm{A}$ beam. The Møller scattered and recoil electrons are detected in coincidence. The collimator system passes electrons Møller scattered near $90^{\circ}$ in the center of mass, where the analyzing power is a maximum, and discriminates against electrons from Mott scattering and other backgrounds. With the target polarization parallel to the beam direction, this polarimeter should be very insensitive to any effects arising from transverse polarization components. The physics analyzing power of the polarimeter determined by Monte Carlo simulation is $A_{z z}=$
$-0.7995 \pm 0.0060$; the uncertainty arises from the statistics of the simulation. The effective analyzing power of the polarimeter is $A_{\text {eff }}=-0.0640 \pm 0.0006$.

\section{EXPERIMENTAL SETUP}

The polarized electron beam is produced by photoemission from a semiconductor cathode using polarized laser light $[14,15]$. The beam polarization depends upon the specific cathode material and the wavelength and degree of polarization of the incident light. The cathode, held at a potential of $-100 \mathrm{kV}$, is a wafer of strained gallium arsenide activated to negative electron affinity. Irradiating the cathode with light of a wavelength slightly shorter than that corresponding to the minimum direct band gap energy of the GaAs produces a beam of highly longitudinally polarized electrons. The longitudinal electron polarization is directly proportional to the circular polarization of the optical beam. It is straightforward to produce optical circular polarization greater than $99 \%$, giving an electron beam longitudinal polarization greater than $70 \%$ from such a cathode. It should be noted that the beam from the photocathode has a pure longitudinal polarization, and that any linear polarization of the illuminating light does not produce transverse electron polarization.

\section{A. Orienting the beam polarization}

The spin rotator is a Wien filter located in the $100 \mathrm{keV}$ beam line following the electron gun. A Wien filter [16] is a static electromagnetic device with electric $(\vec{E})$ and magnetic $(\vec{B})$ fields perpendicular to both the particle velocity $(\vec{\beta})$ and each other as shown in Fig. 6.

The Wien angle $\left(\eta_{\text {Wien }}\right)$ is the angle by which the beam polarization is rotated, in the plane of the electric field, relative to the beam momentum. The Wien angle is directly proportional to the applied fields $\left(\eta_{\text {Wien }}=\eta_{\vec{E}}+\right.$ $\left.\eta_{\vec{B}}\right)$ and at the injector beam energy $(100 \mathrm{keV})$ is dominated by the contribution from the electric field integral:

$$
\frac{\eta_{\vec{B}}}{\eta_{\vec{E}}}=\frac{-a \gamma}{\frac{g}{2 \gamma}-a \gamma}=-0.17 \%,
$$

where the Lorentz factor $\gamma=1.1957$ at $100 \mathrm{keV}, g$ is the electron gyromagnetic factor, and $a=(g-2) / 2=$

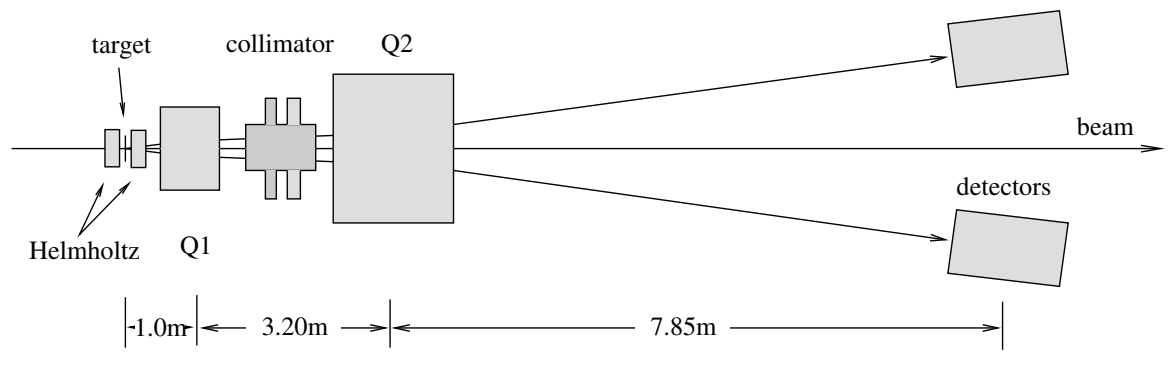

FIG. 5. Schematic of the Hall C Møller polarimeter. 


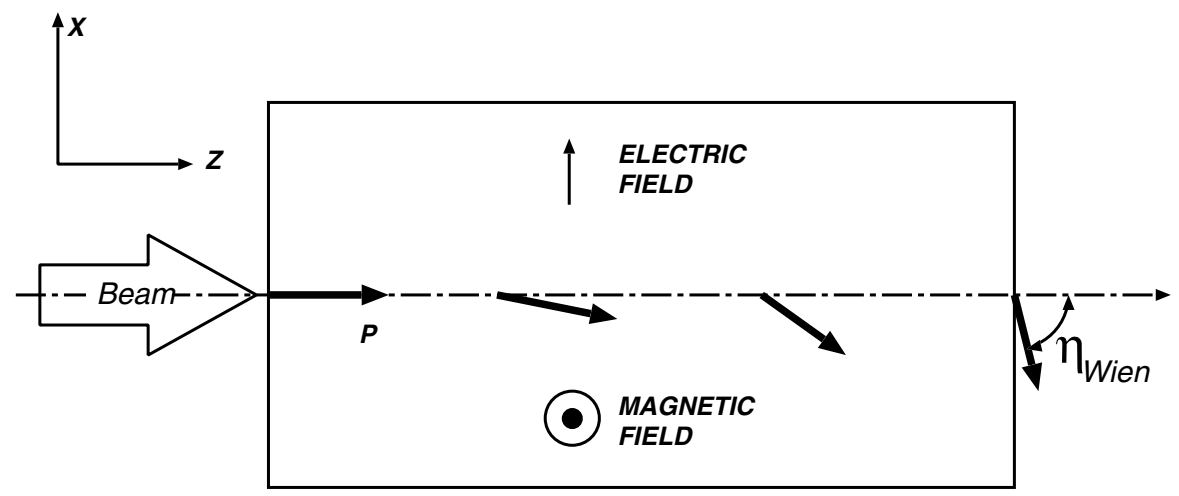

FIG. 6. Diagram of a Wien filter indicating the rotation of the beam polarization relative to the beam direction ( $\left.\eta_{\text {Wien }}\right)$ in crossed magnetic and electric fields $\left(\frac{E}{B}=\beta\right)$. In this experiment, the electric field and the spin rotation are in the horizontal plane.

$1.159652 \times 10^{-3}$. The utility of the Wien filter is that the polarization of a beam passing through the device can be rotated in the plane of the electric field without changing the central beam orbit.

The transverse electric field is produced along the midplane of the Wien filter by two electrodes which span its length. The electrode voltages are set by two opposite polarity $15 \mathrm{kV}$ power supplies controlled by a common digital to analog converter (DAC), so that the potential on axis is zero. A magnetic field normal to the electric field is applied to balance the Lorentz force on the beam axis,

$$
\vec{F}=q(\vec{E}+\vec{\beta} \times \vec{B})=0
$$

requiring that $\left|\frac{E}{B}\right|=\beta$.

The voltage applied across the two electrodes was calibrated against the common DAC setting, with the results shown in Fig. 7. The response is modeled by a second-order polynomial giving the power supply offset, gain, and linearity as a function of the DAC setting

$$
V_{\mathrm{Wien}}= \pm\left(p_{0}+p_{1} \cdot S_{\mathrm{dac}}+p_{2} \cdot S_{\mathrm{dac}}^{2}\right)
$$
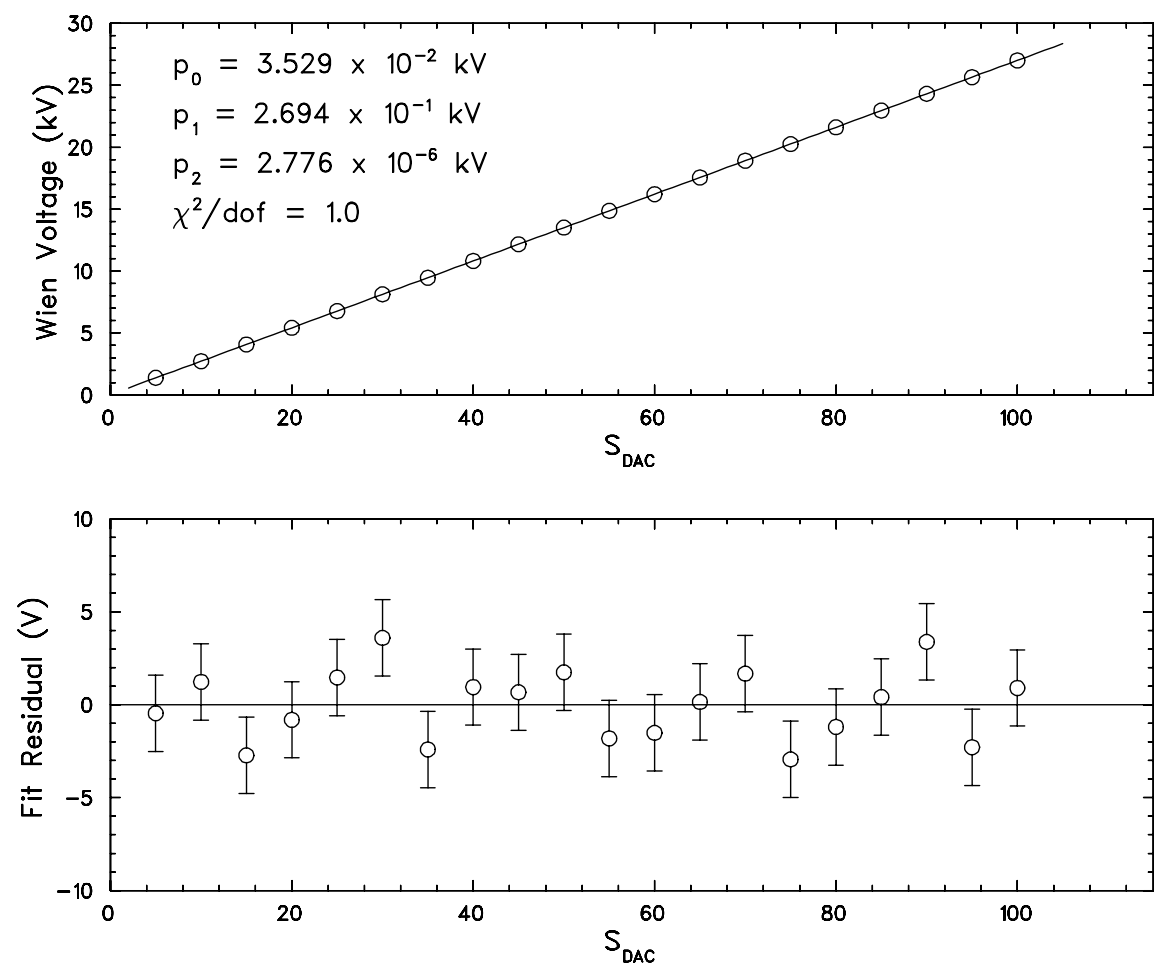

FIG. 7. Wien voltage with second-order polynomial fit (upper panel) and fit residuals (lower panel) both shown as a function of the DAC set point $\left(S_{\text {dac }}\right)$. 
While there is a clear systematic variation in the fit residuals, the magnitude of this effect is much too small to influence any of the results presented here. The overall sign of the spin rotation is set by a high voltage relay that determines which power supply contacts each Wien electrode.

The Wien filter magnetic field is produced with a window-frame dipole magnet. The ends of this magnet are terminated with nickel plates having $2 \mathrm{~cm}$ diameter circular beam apertures. The magnetic field on the axis of the Wien filter, with the nickel plates installed, was carefully measured with a precision Hall probe. The electrodes were shaped to create an electric field profile on the Wien filter axis, as calculated with the code Poisson [17], which very accurately matched the measured magnetic field profile. There was no detectable saturation of the magnet over the full range of field strengths used.

Following the Wien filter, the beam has in general a horizontal polarization component. This component is rotated about the beam axis by any net longitudinal magnetic field integral. Magnetic solenoids are used for beam focusing in several meters of beam line following the Wien filter. Each of these solenoids is comprised of two nominally identical segments oriented to provide equal and opposite longitudinal field integrals, thus giving no net rotation out of the plane to any horizontal polarization component. Any vertical component of polarization exiting the injector arises from imperfect cancellation of the longitudinal field integral of the focusing solenoids acting on the horizontal polarization component exiting the Wien filter and is small.

\section{B. Spin dynamics}

The spin dynamics of the CEBAF accelerator have been extensively studied. Detailed calculations and measurements have demonstrated that the polarization transport is very well understood, and that any loss of beam polarization through the full five-pass accelerator is completely negligible [18].

The magnetic recirculation system, where the vast majority of the spin precession occurs, has no horizontal bends between the equal and opposite vertical bends that take the beam between the two linacs and the various horizontal planes of the recirculation arcs. All bends in each recirculation arc are set in a common horizontal plane by precision survey techniques. This geometry naturally eliminates the difficulties associated with the noncommutation of finite spin rotations which have caused problems in some more complex beam transport systems.

The sensitivity of the final beam polarization to the beam orbit through a single recirculation arc was carefully studied in a dedicated experiment [19]. For this, the spin tune $\left(\nu_{s}=6.4\right)$ and the vertical betatron tune $\left(\nu_{y}=\right.$ $6.0)$ of the arc were close in value. Large equal and opposite vertical betatron oscillations were excited through the arc, and the final polarization value for each orbit measured. The small precessions of the spin in the arc quadrupoles add when the spin tune and the vertical betatron tune are similar, leading to a small but measurable net effect. Detailed simulations of the polarization difference for different orbit pairs, incorporating measured values of the orbits, agreed well with the polarization measurements. For the conditions of the experiment, the longitudinal polarization difference was about $0.5 \%$ per millimeter of absolute orbit displacement. These results show clearly that spin transport through the CEBAF accelerator is very well understood, and that for normal operation of the accelerator, betatron motion in the arcs does not lead to any significant vertical polarization component.

\section{Beam requirements and beam delivery}

The accelerator was configured for five-pass recirculation and a nominal final beam energy of $5.645 \mathrm{GeV}$. The five-pass setup allows delivery of the same energy beam simultaneously to the three end stations. The high beam energy was chosen to minimize the systematic uncertainties for the Møller and Compton polarimeters and to provide the highest analyzing power for the Compton polarimeter.

The polarimeter beam intensity requirements determined how the electron injector was operated. The operational beam intensity required for the five polarimeters varies by 4 orders of magnitude (see Table I). The electron beam produced by a dc gun must ultimately have a bunch structure compatible with the fundamental frequency $(1497 \mathrm{MHz})$ of the accelerator rf. This requirement is assured by an rf chopping system in the $100 \mathrm{keV}$ region of the injector. Three conditions for beam delivery were needed:

(1) solely to the Mott polarimeter at $2 \mu \mathrm{A}$,

(2) solely to the Compton polarimeter above $70 \mu \mathrm{A}$, and

(3) simultaneously to the three Møller polarimeters between $5 \mathrm{nA}$ and $2 \mu \mathrm{A}$.

These conditions were obtained by a combination of choices which deserves some discussion. More than $80 \%$ of a dc electron beam from the polarized source is lost on the apertures of the rf chopping system. A substantial improvement, resulting in a more efficient use of the electrons, has been made by using an $\mathrm{rf}$ driven diode laser [20] providing short optical pulse widths (50 psec FWHM) synchronous with the accelerator rf. Using this laser the emitted electrons have a time structure giving high transmission through the chopping apertures.

There is evidence that the electron polarization depends somewhat on its time within the bunch [21]. This led to the concern that the low beam intensity polarimeters, which used a small fraction of the bunch as defined by the chopping apertures, would receive a beam polarization somewhat different from the high 
beam intensity Compton polarimeter, which used essentially all ( $>98 \%)$ of the bunch.

To address this concern the photoinjector was operated in two different modes. In the first of these, a true dc beam was delivered from the photoemission gun by applying a dc current to the laser. This beam was chopped and bunched in the normal manner, with beam intensity adjustment provided by the chopping apertures. With a dc beam from the polarized gun, the polarization is independent of the time within the bunch. Both Mott and Møller measurements were made with this beam.

The second operating mode was used for the Compton measurements, using the rf driven laser and fully open chopping apertures to reach the higher beam intensity. With the rf driven laser illuminating the cathode, and the fully open chopping apertures, the chopping system passes essentially all of the beam, so there is no issue of beam polarization dependence on time within the bunch. The same laser and optical polarization elements were used for both the dc and the rf cases. Only the electrical drive to the laser was changed between the $\mathrm{dc}$ and the rf cases.

Beam was delivered to the Mott polarimeter alone, using magnetic deflection in the injector, simultaneously to the three Møller polarimeters using rf separation of the high energy beam [22] and to the Compton polarimeter alone by magnetically deflecting the high energy beam.

\section{POLARIMETER MEASUREMENTS AND EXPERIMENTAL RESULTS}

Polarimeter data were collected at 12 different Wien angles spanning $\left|\eta_{\text {Wien }}\right|<110^{\circ}$. The polarimeter data were analyzed by each respective polarimeter group. The measured components of the beam polarization reported by each group are given in Table II.

We report the measured longitudinal polarization for the Møller and Compton polarimeters, and the measured transverse polarization in the horizontal plane for the Mott polarimeter. The measured polarization values are proportional to the product of the longitudinal beam polarization from the photocathode and the cosine of the Wien angle plus the total precession angle.

The measured polarizations from each polarimeter are modeled as

$$
P_{\text {meas }}=P_{i} \cos \left(\lambda_{i} \cdot V_{\text {Wien }}+\Psi_{i}+\phi_{g}\right),
$$

where $P_{i}, \Psi_{i}$, and $\lambda_{i}$ are the fit parameters. $P_{i}$ and $\Psi_{i}$ are the magnitude of the measured beam polarization and the total spin precession between the Wien filter and the particular polarimeter. Although the fit function is periodic, the total beam precession modulo $2 \pi$ is known to much better than one full revolution. Accordingly, the seed value of $\Psi_{i}$ for the fitting routine is set to an appropriate value for each experimental hall. The value $\phi_{g}=a \gamma \theta=-0.01^{\circ}$ is a correction for a single dipole magnet $\left(\theta=-15^{\circ}\right)$ located between the electron source and the Wien filter. The coefficient $\lambda_{i}$ is the constant relating the spin rotation of the Wien filter to the Wien filter high voltage.

The probable values of the fit parameters and their uncertainties are determined from the polarimeter data of Table II using a nonlinear least squares fitting routine [23,24]. Because we are investigating the relative analyzing power of the polarimeters without presuming

TABLE II. Summary of polarization measurements, using the effective analyzing power quoted for each polarimeter. All measurements were made using the dc laser mode except for the Compton measurements which all used the rf laser mode. The uncertainties below are statistical only.

\begin{tabular}{rccccc}
\hline \hline $\begin{array}{c}\eta_{\text {Wien }} \\
(\mathrm{deg})\end{array}$ & $\begin{array}{c}\text { Mott } \\
(\%)\end{array}$ & $\begin{array}{c}\text { Compton A } \\
(\%)\end{array}$ & $\begin{array}{c}\text { Møller A } \\
(\%)\end{array}$ & $\begin{array}{c}\text { Møller B } \\
(\%)\end{array}$ & $\begin{array}{c}\text { Møller C } \\
(\%)\end{array}$ \\
\hline-10.54 & & $-71.05 \pm 0.90$ & $-74.65 \pm 0.17$ & & \\
-10.54 & & $-71.22 \pm 0.85$ & & & \\
57.07 & $-58.90 \pm 0.30$ & & $-36.42 \pm 0.18$ & $-32.65 \pm 1.02$ & $73.62 \pm 0.17$ \\
108.79 & $-69.00 \pm 0.30$ & & $32.09 \pm 0.14$ & $-70.64 \pm 1.02$ & $43.56 \pm 0.26$ \\
93.27 & $-72.80 \pm 0.30$ & $14.41 \pm 1.30$ & $9.45 \pm 0.10$ & $-64.31 \pm 1.61$ & $59.80 \pm 0.27$ \\
77.75 & $-70.60 \pm 0.30$ & $-10.82 \pm 0.61$ & $-12.44 \pm 0.16$ & $-51.97 \pm 0.91$ & $69.50 \pm 0.18$ \\
77.75 & & $-10.81 \pm 0.74$ & & & \\
36.38 & & & $-59.77 \pm 0.16$ & $-8.65 \pm 0.88$ & $67.27 \pm 0.18$ \\
10.54 & $-10.70 \pm 0.40$ & & $-73.02 \pm 0.14$ & $24.05 \pm 0.89$ & $49.37 \pm 0.20$ \\
-10.54 & $13.90 \pm 0.40$ & $-71.52 \pm 1.87$ & $-74.73 \pm 0.14$ & $43.40 \pm 0.99$ & $28.65 \pm 0.26$ \\
-10.54 & & $-78.95 \pm 1.73$ & & & \\
-41.55 & $48.00 \pm 0.40$ & $-58.52 \pm 0.66$ & & & \\
-41.55 & & $-56.71 \pm 0.79$ & & & \\
-60.16 & $62.90 \pm 0.40$ & & $-45.38 \pm 0.13$ & $69.11 \pm 1.02$ & $-30.66 \pm 0.26$ \\
-84.99 & $72.20 \pm 0.20$ & & $-12.39 \pm 0.17$ & $61.95 \pm 1.05$ & $-58.06 \pm 0.22$ \\
-108.79 & $68.00 \pm 0.50$ & & $24.02 \pm 0.14$ & $45.19 \pm 0.99$ & $-72.59 \pm 0.23$ \\
\hline \hline
\end{tabular}


TABLE III. $\lambda_{i}$ determined by Eq. (4) for each polarimeter and the weighted average, $\bar{\lambda}$.

\begin{tabular}{lc}
\hline \hline \multicolumn{1}{c}{ Polarimeter } & $\lambda_{i}(\mathrm{deg} / \mathrm{kV})$ \\
\hline Mott & $5.553 \pm 0.038$ \\
Møller A & $5.666 \pm 0.043$ \\
Møller B & $5.704 \pm 0.047$ \\
Møller C & $5.615 \pm 0.052$ \\
Compton & $5.779 \pm 0.169$ \\
$\bar{\lambda}$ (weighted average) & $5.630 \pm 0.022$ \\
\hline \hline
\end{tabular}

systematic uncertainties, care must be taken to estimate the probable values of the fit parameters and their uncertainties. In doing so we cannot use only the statistical errors reported in Table II because this would underestimate the true variance in a polarimeter data set. The approach taken $[24,25]$ is to assume that we do not know the individual measurement errors and to assign to each measurement in a polarimeter data set an equal unit weight error. Consequently, the standard error of estimate for the fit parameters from each data set is given by the root mean square statistical errors of estimate of the covariance matrix, weighted by the reduced chi square of the fit $\sqrt{\chi^{2} /(N-M)}$. Here $\chi^{2}$ is the least squares estimate evaluated at the most probable fit parameters, $N$ is the number of measurements in a data set, and $M$ is the number of fit parameters. This approach depends upon the following assumptions:

(i) we are using the correct model to describe the data [see Eq. (4)],

(ii) the systematic errors do not change within a data set, and

(iii) the total measurement error of each data point within a data set is approximately equal, requiring that each measurement has a very similar statistical precision.

While the first and third assumptions are valid, it is important to note that the systematic uncertainty may depend on the degree of horizontal polarization for some polarimeters, so the second assumption may not be met in all cases. We have not attempted to analyze how this might affect our results.

To compare the polarimeters one must additionally constrain the parameter $\lambda_{i}$ to be equal for all polarimeter data measured at identical Wien angle settings. Consequently, the analysis was segmented into two stages. In the first stage all three fit parameters are estimated and the weighted average $(\bar{\lambda})$ of the set of $\lambda_{i}$ (see Table III) is computed. In the second stage each data set was fit again using Eq. (4) with $\lambda_{i}$ replaced by the weighted average value $\bar{\lambda}$. The first stage analysis of the data gives a $\lambda_{i}$ that varies by $4.0 \%$ between the extremes of the polarimeter data sets. This variation is attributed to an unmeasured systematic effect and is included in the uncertainty of the fit. Although unexpected, this variation has little impact on the final determination of the beam polarization and spin phase, as demonstrated below.

The final fit results and residuals are shown in Fig. 8. In Table IV we compare the results obtained for $P_{i}$ and $\Psi_{i}$ for each polarimeter using both the individual $\lambda_{i}$ for that polarimeter and using $\bar{\lambda}$. In all cases the differences in the fit results using either $\lambda_{i}$ or $\bar{\lambda}$ are small compared to the standard error from the least squares fitting routine.

It is important to understand that the systematic uncertainties associated with each polarimeter are not included in the polarization results given in Table IV and Fig. 8. A motivation for this experiment is to find evidence of undetected systematic effects or other problems with the quoted effective analyzing powers of the various polarimeters by comparing the polarization determined by each polarimeter when all measure the same polarized beam. When systematic effects are included, the uncertainties on the polarization values reported in Table IV and Fig. 8 will be larger.

A comparison of the beam polarizations determined from each polarimeter data set is made in Fig. 9 by plotting the ratio of the measured polarization values $\left(P_{i}\right)$ with respect to a reference. In this case, the Mott polarimeter was chosen as the reference because it had the smallest relative uncertainty in the fit parameter $P_{i}$. The ratio of the effective analyzing power of any polarimeter relative to the Mott polarimeter is the inverse of the ratio shown in Fig. 9.

As noted earlier, when the longitudinal polarization component is small, the horizontal component is large. Some polarimeters may have systematic effects that depend on the horizontal component. An indication of the

TABLE IV. Polarization and phase results for polarimeter data using $\bar{\lambda}=5.630^{\circ} / \mathrm{kV}$; italicized text show the values obtained using the individual $\lambda_{i}$. The uncertainties are the total standard error in the fit parameters using the least squares fitting routine.

\begin{tabular}{lccrr}
\hline \hline & \multicolumn{2}{c}{ Polarization $(\%)$} & \multicolumn{2}{c}{ Phase $(\mathrm{deg})$} \\
Polarimeter & $\bar{\lambda}$ & $\lambda_{i}$ & \multicolumn{1}{c}{$\lambda_{i}$} \\
\hline Mott & $72.10 \pm 0.28$ & $72.19 \pm 0.24$ & $88.88 \pm 0.32$ & $88.82 \pm 0.27$ \\
Møller A & $76.76 \pm 1.04$ & $76.79 \pm 1.06$ & $10983.84 \pm 0.63$ & $10983.75 \pm 0.64$ \\
Møller B & $69.84 \pm 0.64$ & $69.81 \pm 0.59$ & $10500.48 \pm 0.56$ & $10500.30 \pm 0.53$ \\
Møller C & $73.30 \pm 0.62$ & $73.29 \pm 0.66$ & $10023.39 \pm 0.61$ & $10023.40 \pm 0.65$ \\
Compton & $73.33 \pm 1.42$ & $74.25 \pm 1.84$ & $10984.82 \pm 1.33$ & $10983.07 \pm 2.36$ \\
\hline \hline
\end{tabular}



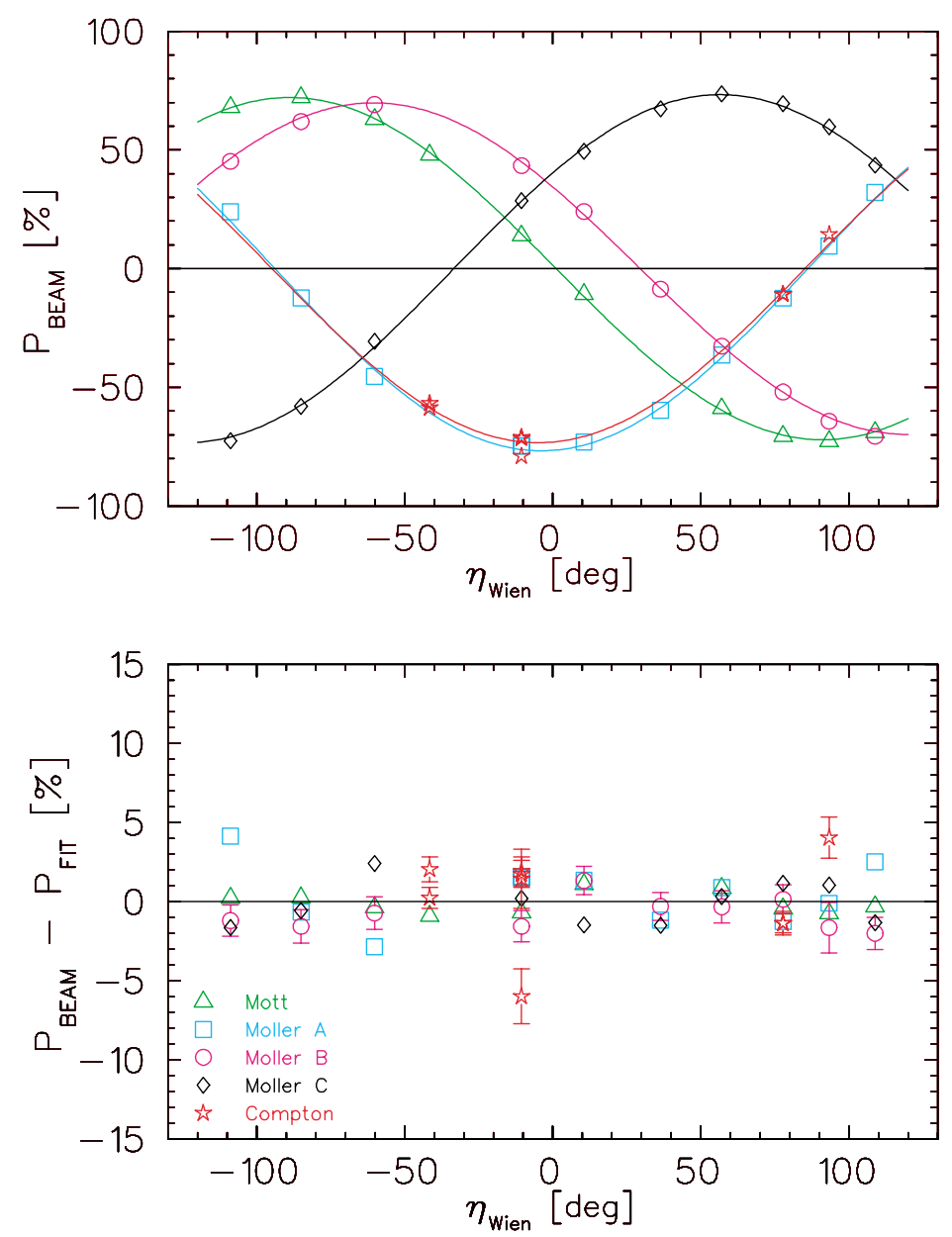

FIG. 8. (Color) Measured polarizations and fits (upper plot) using $\bar{\lambda}$ and all data. Fit residuals (lower plot), with only statistical uncertainties from the fits shown. In general, the uncertainties from the fits are smaller than the symbol sizes used in plotting the data. Note: the lower plot legend applies to the upper plot.

importance of this effect may be had by fitting Eq. (4) to data sets containing only large longitudinal polarization values. Figure 10 and Table V show the results of fits where the data fitted have been restricted to be within $25 \%$ of the maximum polarization value. Such fits do not give as good values for $\Psi_{i}$, but still give good values for $P_{i}$. The ratio of these polarization values to the Mott polarization value is given in Fig. 11. These results indicate that the

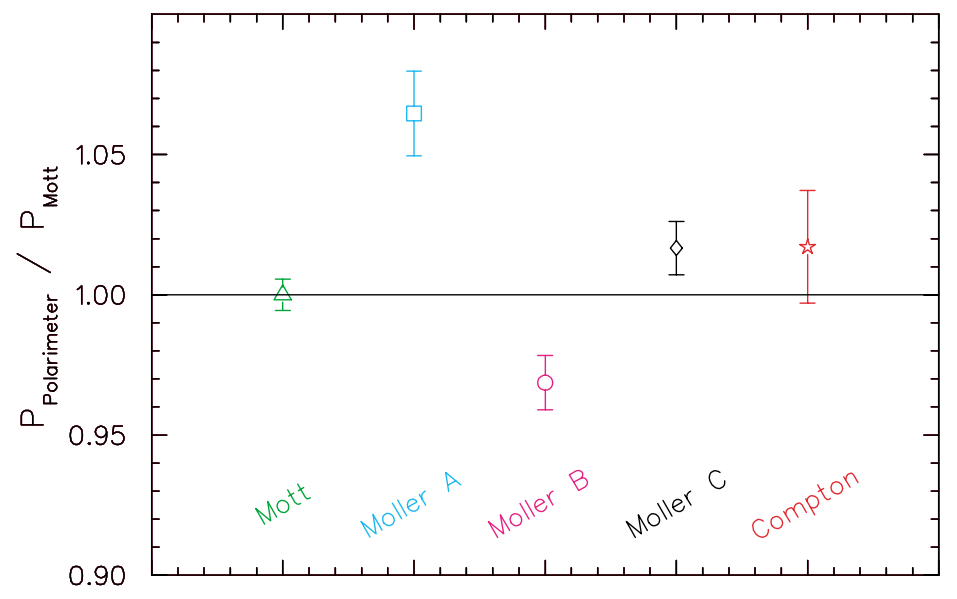

FIG. 9. (Color) The relative analyzing powers for the five Jefferson Laboratory electron beam polarimeters, normalized to the Mott polarimeter for comparison. 

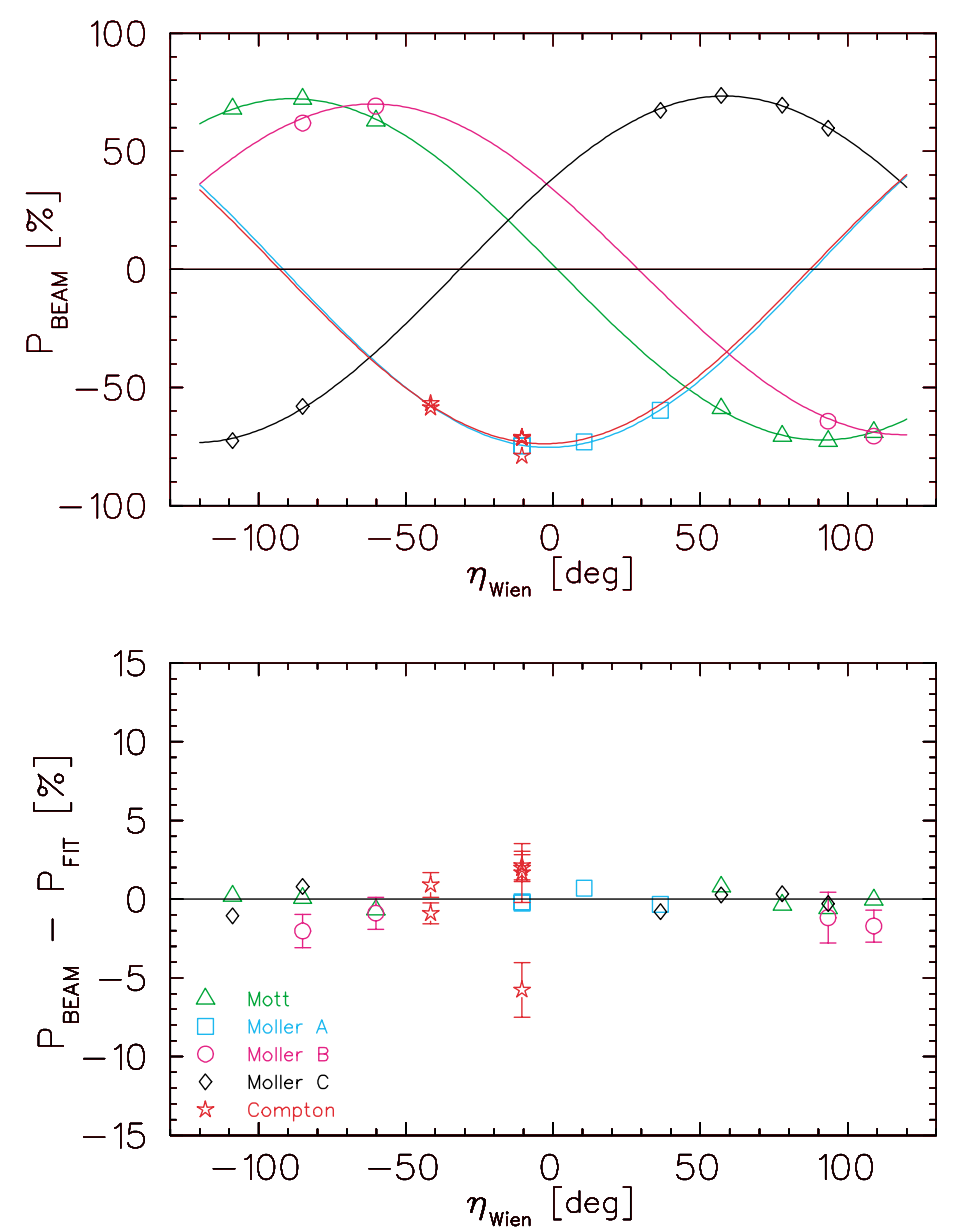

FIG. 10. (Color) Polarimeter data and fit (upper plot) using $\bar{\lambda}$ and data set limited to be within $25 \%$ of the maximum polarization. Fit residuals (lower plot) are shown using only statistical uncertainties from polarimeter data. Note: the lower plot legend applies to the upper plot.

horizontal component of polarization may be an important source of systematic effects for the Hall A Møller polarimeter.

\section{BEAM ENERGY MEASUREMENTS}

The beam energy is usually measured by either high precision magnetic spectrometer or elastic electron- proton scattering methods. An additional benefit of the polarimeter intercomparison is that the spin precession data can be used for a precision determination of the beam energy, due to the linear relationship between the spin precession and the beam energy. One of two independent spin-based approaches, described below, is shown to be capable of yielding an extremely precise absolute measurement $\left(<10^{-4}\right)$ of the beam energy.

TABLE V. Polarization and phase results for polarimeter data restricted to be within $25 \%$ of the maximum polarization measured; italicized text show results using all data (see Table IV) for comparison. The uncertainties are the total standard error in the fit parameters using the least squares fitting routine. Results presented in this table use $\bar{\lambda}=5.630^{\circ} / \mathrm{kV}$.

\begin{tabular}{lccrr}
\hline \hline & \multicolumn{2}{c}{ Polarization $(\%)$} & \multicolumn{2}{c}{ Phase $(\mathrm{deg})$} \\
Polarimeter & $25 \%$ restriction & \multicolumn{1}{c}{ All data } & 25\% restriction & \multicolumn{1}{c}{ All data } \\
\hline Mott & $72.27 \pm 0.23$ & $72.10 \pm 0.28$ & $88.59 \pm 0.50$ & $88.88 \pm 0.32$ \\
Møller A & $75.40 \pm 0.33$ & $76.76 \pm 1.04$ & $10981.60 \pm 0.68$ & $10983.84 \pm 0.63$ \\
Møller B & $70.01 \pm 1.80$ & $69.84 \pm 0.64$ & $10501.07 \pm 4.54$ & $10500.48 \pm 0.56$ \\
Møller C & $73.37 \pm 0.42$ & $73.30 \pm 0.62$ & $10021.70 \pm 0.74$ & $10023.39 \pm 0.61$ \\
Compton & $73.85 \pm 2.15$ & $73.33 \pm 1.42$ & $10982.86 \pm 4.10$ & $10984.82 \pm 1.33$ \\
\hline \hline
\end{tabular}




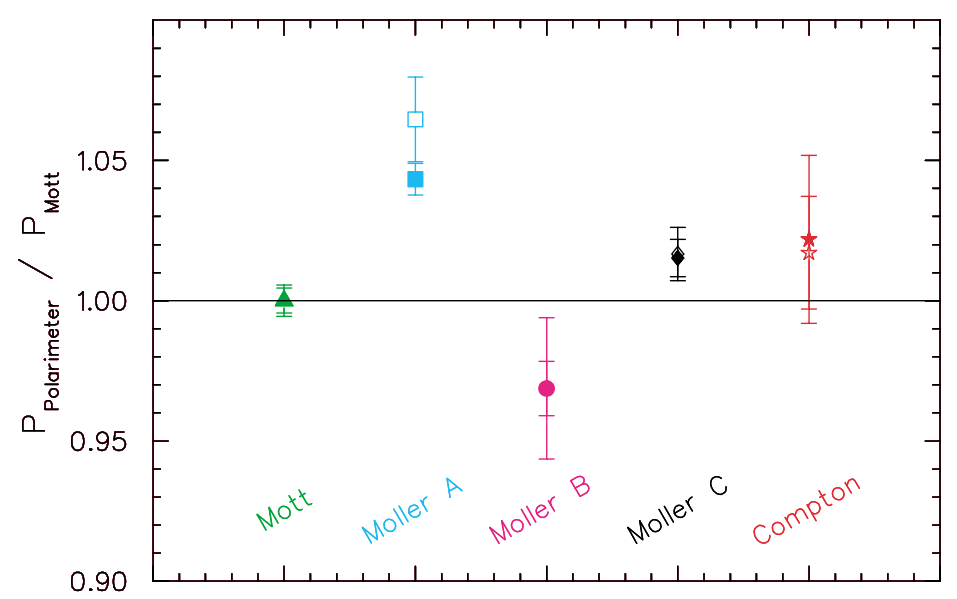

FIG. 11. (Color) The relative analyzing powers for the five Jefferson Laboratory electron beam polarimeters, normalized to the Mott polarimeter for comparison. The solid symbol markers represent the results for the data set limited to be within $25 \%$ of the maximum measured polarization. The open symbol markers are the results shown in Fig. 9.

For comparison, the beam energy was measured using the magnetic spectrometer method [26]. Two pairs of beam profile monitors measured the beam direction before and after a string of eight well-measured dipole magnets leading into Hall A to determine the resulting beam deflection. This measurement gave a five-pass beam energy of $5646.5 \pm 3.0 \mathrm{MeV}$.

\section{A. Method No. 1: Beam energy measured by spin precession between the injector and the experimental halls}

The electron beam gains an initial energy in the injector. After injection into the main accelerator the electron beam successively gains energy in each linac during each recirculation pass (see Fig. 1). The dipole magnets in the recirculation and experimental hall transport arcs precess the beam polarization. The total spin precession between the injector and any experimental hall, as measured by the Mott polarimeter and the corresponding experimental hall polarimeter, can be exactly calculated. For $n$ recirculations through the accelerator $(n=5$ for this experiment) the total precession, $\Psi_{n}$, can be summed and written, after some algebraic manipulation, as

$$
\begin{aligned}
\Psi_{n}=\left(\frac{g-2}{2 m_{e}}\right)\{ & \left\{n \theta_{1}+(n-1) \theta_{2}\right] E_{0} \\
& +\frac{n}{2}\left[(n+1) \theta_{1}+(n-1) \theta_{2}\right] E_{1} \\
& +\frac{n(n-1)}{2}\left(\theta_{1}+\theta_{2}\right) E_{2} \\
& \left.+\left[E_{0}+n\left(E_{1}+E_{2}\right) \theta_{h}\right]\right\},
\end{aligned}
$$

where $E_{0}, E_{1}$, and $E_{2}$ are the energy gains of the injector, north linac and south linac, $\theta_{1}$ and $\theta_{2}$ are the bend angles of the east and west recirculation arcs, and $\theta_{h}$ is the bend angle of the respective experimental hall transport arc $(h \in\{\mathrm{A}, \mathrm{B}, \mathrm{C}\}$ ). Note that Eq. (5) assumes that the energy gain on each pass through each linac is the same. In practice, this is assured by measuring and correcting the total path length of each recirculation pass. The system developed to do this allows the path length on each pass to be set with a $2 \sigma$ precision of better than 0.25 rf degree, leading to negligible differences in the energy gain on each pass [27]. It is useful to transform Eq. (5) to parameters more practical (see Table VI) for evaluating the beam energy.

After manipulation, the final beam energy is written in terms of these accelerator parameters and the total spin precession determined from the polarimeter measurements as

$$
E=\frac{\frac{4 m_{e} \Psi_{n}}{g-2}-E_{0}\left(\theta_{t}-\theta_{h}\right)-\frac{n E_{12}\left[\theta_{t}-\theta_{h}+(n-1) \theta_{12}\right]}{(2 n-1)}}{\theta_{t}+\theta_{h}} .
$$

The main advantage of this method is that at the highest CEBAF energies one can take advantage of the very large total precession $\left(\Psi>10000^{\circ}\right)$ to reach an absolute measurement of the beam energy to better than $10^{-4}$. To do so requires precise knowledge of the accelerator parameters in Eq. (6). The sensitivity of the beam energy to these parameters is given in Table VII and is described in more detail below.

Uncertainty in the injector beam energy $\left(E_{0}\right)$ is a significant contribution to the total uncertainty because this fraction of the beam energy is precessed by each

TABLE VI. Transformations to practical accelerator parameters.

\begin{tabular}{lc}
\hline \hline \multicolumn{1}{c}{ Quantity } & Transformation \\
\hline Final beam energy & $E_{0}+n\left(E_{1}+E_{2}\right) \rightarrow E$ \\
Linac imbalance & $E_{1}-E_{2} \rightarrow E_{12}$ \\
Total bend angle & $n \theta_{1}+(n-1) \theta_{2}+\theta_{h} \rightarrow \theta_{t}$ \\
Linac skewness & $\theta_{1}-\theta_{2} \rightarrow \theta_{12}$ \\
\hline \hline
\end{tabular}


TABLE VII. The derivative of Eq. (6) with respect to each of the dependent parameters is shown alongside the corresponding the numerical value calculated for Hall $\mathrm{B}$. The numerical values for Halls A and $\mathrm{C}$ are within $5 \%$ of the values for Hall B.

\begin{tabular}{cc}
\hline \hline Derivative & Numerical value \\
\hline$\left(\partial E / \partial \Psi_{n}\right)=\left[4 m_{e} /(g-2)\right] /\left(\theta_{t}+\theta_{h}\right)$ & $0.544 \mathrm{MeV} / \mathrm{deg}$ \\
$\left(\partial E / \partial E_{0}\right)=-\left(\theta_{t}-\theta_{h}\right) /\left(\theta_{t}+\theta_{h}\right)$ & $-1.00 \mathrm{MeV} / \mathrm{MeV}$ \\
$\left(\partial E / \partial \theta_{12}\right)=-\left[n(n-1) E_{12} /(2 n-1)\right] /\left(\theta_{t}+\theta_{h}\right)$ & $-0.00713 \mathrm{MeV} / \mathrm{deg}$ \\
$\left(\partial E / \partial E_{12}\right)=-n\left\{\left[\theta_{t}-\theta_{h}+(n-1) \theta_{12}\right] /\left[(2 n-1)\left(\theta_{t}+\theta_{h}\right)\right]\right\}$ & $-0.556 \mathrm{MeV} / \mathrm{MeV}$ \\
$\left(\partial E / \partial \theta_{t}\right)=\left[-E-E_{0}-n E_{12} /(2 n-1)\right] /\left(\theta_{t}+\theta_{h}\right)$ & $-3.527 \mathrm{MeV} / \mathrm{deg}$ \\
$\left(\partial E / \partial \theta_{h}\right)=\left[-E+E_{0}+n E_{12} /(2 n-1)\right] /\left(\theta_{t}+\theta_{h}\right)$ & $-3.449 \mathrm{MeV} / \mathrm{deg}$ \\
\hline \hline
\end{tabular}

dipole magnet of the accelerator. The injector beam momentum was measured immediately prior to the experiment using a recent high precision calibration of the injector spectrometer [28]. This gives a value for the injector beam energy of $E_{0}=62.89 \pm 0.06 \mathrm{MeV}$.

The precision with which the bend angles of the recirculation $\operatorname{arcs}\left(\theta_{1}, \theta_{2}\right)$ and hall transport $\operatorname{arcs}\left(\theta_{h}\right)$ is known also impacts the energy measurement. These angles have been determined by high precision survey measurements of beam line elements in the linacs and at the beginning and end of the transport beam lines into experimental Halls A and C. The survey measurements are made with respect to the accelerator site reference grid, which in turn is established within a network of survey monuments spanning the accelerator site.

At the time of the measurements reported here, gyrotheodolite measurements [29] were made at a number of locations throughout the accelerator site to confirm the overall shape and stability of the accelerator site reference grid. A gyrotheodolite is an instrument that measures, with very high precision (ca. 3 arc sec), the horizontal direction of a line with respect to true north - the Earth's axis of rotation. The gyrotheodolite was used to transfer a common high precision directional reference to locations throughout the accelerator tunnel complex. One can then compare the azimuths as determined from the gyrotheodolite measurements to azimuths calculated from precision survey measurements. There are three sources of measurement uncertainty in determining these angles: (a) the 3 arc sec uncertainty of the gyrotheodolite; (b) an azimuthal uncertainty resulting from the transverse position measurement uncertainty of $0.25 \mathrm{~mm}$ and the separation of the beam line elements; and (c) an azimuthal uncertainty resulting from the transverse component of the fiducialization uncertainty of $0.05 \mathrm{~mm}$ and the separation of the beam line elements. Using normal error propagation, these differences in azimuth were less than $1.5 \mathrm{arcsec}$ in the linacs, approximately $10 \mathrm{arcsec}$ in the beam switchyard, less than 5 arc sec in the Hall A transport line, and less than 10 arc sec in the Hall $\mathrm{C}$ transport line. Overall, the gyrotheodolite measurements verify the accelerator site reference grid at the level of the 3 arc sec gyrotheodolite measurement uncertainty and justify the use of precision survey measurements to determine the angles necessary for the energy determination. The angles used are given in Table VIII.

Finally, the polarization precession depends on the linac energy imbalance, $E_{12}$, which is the difference in the energy gain between the north and the south linacs. Consider the case in which the energy gained in one full recirculation pass is fixed. The west recirculation arcs follow both linacs, so the precession in these arcs does not depend on the equality of the linac energy gains. However, the east recirculation arcs follow only the north linac, and thus the precession in these arcs depends on $E_{12}$. In particular, precession in the east arcs is greater when $E_{12}>0$.

$E_{12}$ was not measured at the time of the precession measurements reported here. Instead, this measurement was made soon afterward, when the energy gain in each linac had been increased a nominal $7 \%$. To measure $E_{12}$ a single pass beam was sent to Hall A, and its energy was determined by the standard method of measuring the total deflection through the eight Hall A transport dipoles. The beam energy was measured with both linacs powered, and with only the north linac powered and the beam simply drifted through the unpowered south linac.

TABLE VIII. The gyrotheodolite survey measurements are shown along with the resulting calculated parameters needed for the spin precession results. The angle of the beam line to Hall B was not surveyed with the gyrotheodolite and is included as an assumed value.

\begin{tabular}{ccr}
\hline \hline Quantity & Acquired & \multicolumn{1}{c}{ Angle (deg) } \\
\hline$\theta_{h=\mathrm{A}}$ & Measured & $37.4913 \pm 0.0020$ \\
$\theta_{h=\mathrm{B}}$ & Assumed & $0.0000 \pm 0.0100$ \\
$\theta_{h=\mathrm{C}}$ & Measured & $-37.4774 \pm 0.0057$ \\
$\theta_{1}$ & Measured & $180.0000 \pm 0.0020$ \\
$\theta_{2}$ & Measured & $180.0000 \pm 0.0020$ \\
$\theta_{12}$ & Calculated & $0.0000 \pm 0.0020$ \\
$\theta_{t=\mathrm{A}}$ & Calculated & $1657.4913 \pm 0.0024$ \\
$\theta_{t=\mathrm{B}}$ & Calculated & $1620.0000 \pm 0.0101$ \\
$\theta_{t=\mathrm{C}}$ & Calculated & $1582.5226 \pm 0.0058$ \\
\hline \hline
\end{tabular}


TABLE IX. Summary of measurements to determine the linac energy imbalance.

\begin{tabular}{lcc}
\hline \hline \multicolumn{1}{c}{ Quantity } & Acquired & Beam energy $(\mathrm{MeV})$ \\
\hline Injector & Measured & $67.89 \pm 0.07$ \\
North linac only & Measured & $671.14 \pm 0.28$ \\
North and south linac & Measured & $1270.26 \pm 0.52$ \\
$E_{1}$ & Calculated & $603.25 \pm 0.29$ \\
$E_{2}$ & Calculated & $599.12 \pm 0.59$ \\
\hline \hline
\end{tabular}

The results are reported in Table IX. These measurements gave a value of $4.13 \pm 0.66 \mathrm{MeV}$ for $E_{12}$ at this energy.

The value of $E_{12}$ at the energy used for the precession measurements was determined by scaling to the lower energy, using accelerator parameters measured at each energy. Two different methods were used to do this scaling. In the first of these, the sum of the accelerating gradients of the 160 cavities in each linac was compared at the two beam energies. In the second, detailed measurements of the beam orbit through the first and second recirculation arcs, coupled with measurements of the dipole bus current in these arcs, were used. These two scaling methods gave values for $E_{12}$ of $4.75 \pm 0.76 \mathrm{MeV}$ and $5.69 \pm 0.95 \mathrm{MeV}$, respectively. The weighted mean of these two values is $5.17 \pm 0.59 \mathrm{MeV}$. For the analysis presented here, we use the value $E_{12}=5.2 \pm 1.0 \mathrm{MeV}$. The enlarged uncertainty in the value of $E_{12}$ is chosen to account for any undetected systematic effects associated with determining $E_{12}$ at a different time and with different linac energy gains.

With the above value for $E_{12}$, we can determine four values for the final beam energy from the measurements of the total precession to the four hall polarimeters. These values are presented in Table $\mathrm{X}$. The total five-pass pre-

TABLE $X$. Final beam energies measured by total precession evaluated at $E_{12}=5.2 \pm 1.0 \mathrm{MeV}$.

\begin{tabular}{ccc}
\hline \hline Polarimeters & $\Psi(\mathrm{deg})$ & $E(\mathrm{MeV})$ \\
\hline Mott-Compton & $10985.94 \pm 1.37$ & $5649.21 \pm 0.89$ \\
Mott-Møller A & $10984.96 \pm 0.71$ & $5648.70 \pm 0.65$ \\
Mott-Møller B & $10501.60 \pm 0.64$ & $5647.20 \pm 0.66$ \\
Mott-Møller C & $10024.51 \pm 0.69$ & $5649.03 \pm 0.71$ \\
\hline \hline
\end{tabular}

cession for each polarimeter is that listed in Table IV, corrected by the polarization orientation as measured in the injector by the Mott polarimeter $\left(1.12^{\circ} \pm 0.32^{\circ}\right)$.

\section{B. Method No. 2: Beam energy measured by relative spin precession differences between experimental halls}

The alternative to comparing the total precession between the injector and an experimental hall polarimeter is simply to use the precession difference between each experimental hall. The advantage is that prior to extraction, the beam to each hall undergoes identical precession. In this way the injector energy, linac imbalance, and recirculation arc bend angles are eliminated from the equation and the precession between any two experimental hall polarimeters is given simply by

$$
\Delta \Psi=\frac{g-2}{2} \frac{E}{m_{e} c^{2}} \cdot \Delta \Theta
$$

where $\Delta \Psi$ and $\Delta \Theta$ are the measured differences in the precession and bend angle between two respective hall polarimeters. $E$ is the final beam energy common to both. In this case, the uncertainty in the bend angle is the main contribution to the energy uncertainty. The disadvantage of this method is that the precession difference between different experimental hall polarimeters is much smaller than the precession through the full five-pass accelerator. Thus, even at the maximum CEBAF energy, the statistical precision of this energy measurement is of order $10^{-3}$. The beam energies obtained from the precession differences between the four experimental hall polarimeters are given in Table XI.

\section{Final energy determination}

Both the injector to experimental hall and experimental hall to experimental hall spin-based energy measurement methods indicate discrepancies associated with the Hall B polarimeter. The energy as determined by the precession from the injector to the Hall B polarimeter is somewhat in disagreement $(>2 \sigma)$ with the energy determined with the three other polarimeters, all of which are themselves in good agreement. The energies determined from the precession difference between the Hall A and Hall C polarimeters are consistent with one

TABLE XI. Summary of energy measurement results comparing only end-station polarimeters by the relative spin precession method.

\begin{tabular}{lcccc}
\hline \hline \multicolumn{1}{c}{ Polarimeters } & $\Delta \Psi(\mathrm{deg})$ & \multicolumn{1}{c}{$\Delta \Theta(\mathrm{deg})$} & \multicolumn{1}{c}{$E(\mathrm{MeV})$} & $\frac{\sigma_{E}}{E}(\%)$ \\
\hline Møller A-Møller B & $483.36 \pm 0.84$ & $37.4913 \pm 0.0102$ & $5681.10 \pm 10.03$ & 0.176 \\
Møller A-Møller C & $960.45 \pm 0.88$ & $74.9687 \pm 0.0060$ & $5645.30 \pm 5.17$ & 0.092 \\
Compton A-Møller B & $484.34 \pm 1.44$ & $37.4913 \pm 0.0102$ & $5692.62 \pm 17.03$ & 0.299 \\
Compton A-Møller C & $961.43 \pm 1.46$ & $74.9687 \pm 0.0060$ & $5651.07 \pm 8.61$ & 0.152 \\
Møller B-Møller C & $477.09 \pm 0.83$ & $37.4774 \pm 0.0115$ & $5609.49 \pm 9.89$ & 0.176 \\
\hline \hline
\end{tabular}


another and consistent with the much more precise values determined by the total precession method. However, there are large and statistically untenable discrepancies in the energies determined by using the precession differences between the Hall B polarimeter and the Hall A and $\mathrm{C}$ polarimeters. When comparing either the Hall $\mathrm{A}$ or Hall C polarimeter to the Hall B polarimeter these discrepancies, about $37 \mathrm{MeV}$ (see Table XI), clearly indicate that the difference is associated with whether the comparison polarimeter is located in Hall A (higher energy) or Hall C (lower energy).

Inspection of Eq. (7) shows that the discrepancies associated with the Hall B polarimeter can be resolved by either increasing the total precession from the injector to the Hall B polarimeter, $\Psi$, by $3.4^{\circ}$ or an angular shift of the Hall B beam line, $\theta_{h=\mathrm{B}}$, by $0.26^{\circ}$ further from Hall $\mathrm{A}$ and closer to Hall $\mathrm{C}$.

Inserting these two possibilities into Eq. (6) for the energy determined from the total spin precession method yields a beam energy of $5649.05 \pm 0.66$ for the modified precession or $5648.10 \pm 0.66$ for the modified beam line. In either case, these new values agree better with the energy as determined by the total precession to the Halls $\mathrm{A}$ and $\mathrm{C}$ polarimeters.

It is not possible to determine the source of the discrepancy associated with the Hall B polarimeter energy determination from our measurements. The required shift in precession of about $3.4^{\circ}$ is very large compared to the standard deviation of the fit value for the Hall $\mathrm{B}$ total precession $\left(0.64^{\circ}\right)$, making a precession error this large unlikely. The angle of the beam line into Hall B was not determined as part of the precision gyrotheodolite survey, leaving open the possibility that there is a small angular misalignment of the polarimeter beam line. Further measurements are required to resolve the present discrepancy.

At this point, we choose to ignore all energy measurements involving the Hall B polarimeter. We can determine a best value for the full five-pass accelerator energy from the three independent measurements of the total precession from the injector to the two Hall A and one Hall C polarimeters. The statistically weighted energy as determined from these three measurements is $5648.94 \pm$ $0.42 \mathrm{MeV}$. This value is within less than 1 standard deviation of the value of $5646.5 \pm 3.0 \mathrm{MeV}$ as determined by the magnetic spectrometer method.

\section{CONCLUSIONS}

A careful comparison of the relative analyzing powers of the five Jefferson Laboratory electron polarimeters, based on Mott, Møller, and Compton scattering, has been performed. This was accomplished by using a Wien filter spin rotator located in the low energy region of the injector to orient the electron spin between $\pm 110^{\circ}$ relative to the beam direction in the horizontal plane.
Asymmetry measurements as a function of this spin orientation were conducted with each polarimeter. Since each polarimeter measures a beam with the same magnitude of polarization, a determination of the relative analyzing power of the various polarimeters is straightforward. Furthermore, a measurement of the spin orientation, as set by the Wien filter, that produces maximum longitudinal polarization at each polarimeter leads to a very high precision energy measurement. At the accelerator energies employed in this experiment, measurement of the total precession from the injector to a single polarimeter leads to a determination of the beam energy with a precision of about $10^{-4}$.

We make the following observations with respect to our results.

(i) Polarimeters: It is clear from these results that the five Jefferson Lab polarimeters do not all agree on the measured beam polarization at the level of a few percent. The effective analyzing power of each of these polarimeters is a simulated number. Polarimeter comparisons of this type help to reveal undetected systematic effects and other sources of error in the simulated values of the effective analyzing powers. A thorough understanding of polarimeter systematic effects will be necessary to achieve absolute electron polarimetery at the $1 \%$ level, as is required for some proposed parity violation measurements. While precision comparisons of polarimeter effective analyzing powers can never guarantee that the effective analyzing powers used are correct, agreement among the values of the beam polarization as measured by very different polarimeters provides strong circumstantial evidence that no large systematic effects have been overlooked. In general, our results indicate how difficult it will be to establish a convincing case that a polarimeter is capable of $1 \%$ absolute electron polarization measurements.

The polarimeter comparison presented here is interesting in that we have reported measurements of the longitudinal polarization component only. In all cases, these measurements were made with a highly polarized beam. Thus, when the measured longitudinal component is small there is necessarily a large transverse component in the horizontal plane for these measurements. The presence of a large transverse spin component may result in systematic effects in the effective longitudinal analyzing power of a particular polarimeter. Our measurements indicate that large transverse spin components may be an important source of systematic effects for conventional Møller polarimeters.

Finally, it is worth noting that the beam polarizations determined by the injector Mott polarimeter, the Hall A Compton polarimeter, and the Hall C Møller polarimeter are in reasonable statistical agreement with a single value. Note that the errors on the values given in Table IV are from the fit only and do not include either the measurement statistics or the systematic uncertainty estimated for 
each polarimeter. The actual uncertainties in the measured polarization values are larger than those shown in Table IV. This result implies that the analyzing powers of these three very different polarimeters are reasonably well understood.

(ii) Wien filter: The value of the Wien angle coefficient $\lambda$, as determined from fits of the longitudinal polarization measured with each polarimeter as a function of the Wien angle, depends on the particular polarimeter used. While this effect is not understood, the resulting uncertainty in the measured analyzing power and precession angle due to the uncertainty in the value of $\lambda$ is small. Two data sets, taken with the injector Mott polarimeter about one month apart, gave fit values for $\lambda$ differing by about $1 \%$. Precision measurement of the gap high voltage and the magnet current associated with each measurement, as well as the magnet hysteresis history, is clearly warranted. Operationally, the beam orbit through the Wien filter may be slightly different with different Wien filter settings, particularly as the Wien angle becomes large. While this systematic effect does not affect the conclusions from the measurements reported here, it should be better understood for future polarimeter comparison measurements.

(iii) Energy measurements: The total precession energy measurement using only the Hall A and Hall C polarimeters yields a statistically weighted mean, from three independent measurements, of $5648.94 \pm 0.42 \mathrm{MeV}$, or a precision of $0.74 \times 10^{-4}$. The value is well within 1 standard deviation of the magnetic arc spectrometer measurement of $5646.5 \pm 3.0 \mathrm{MeV}$. The systematic uncertainties of the magnetic spectrometer measurement are very different than the systematic uncertainties of the total precession measurements. The statistically weighted mean of these two total energy measurements is 5648.89 \pm $0.42 \mathrm{MeV}$. A major source of uncertainty in the present total precession measurements is the linac energy imbalance. The value used in the above measurements includes an enlarged uncertainty to account for the fact that the energy imbalance was not measured at the same time, or at the exact energy, of the spin-based energy measurements. This source of uncertainty could be greatly reduced in a future spin-based energy measurement. There is clear evidence of problems with the energy measurements based on the Hall B polarimeter, and some evidence of a misalignment of the beam to the Hall B polarimeter.

Jefferson Lab is developing high precision electron polarimetry to meet the needs of future experiments. A comparison of the beam polarization measured by different polarimeters observing the same beam is an important way to gain confidence that the effective analyzing powers of these polarimeters have been correctly determined. The results presented here clearly demonstrate that measurements of the total precession through the CEBAF accelerator can be used to determine the absolute value of the accelerator energy with exceptional precision. While spin-based energy measurements are too cumbersome for routine use, a program of such measurements could provide useful high precision cross calibrations of more easily applied energy measurement techniques.

\section{ACKNOWLEDGMENTS}

This work is the result of contributions by many scientists dedicated to producing and measuring the polarized electron beam at Jefferson Laboratory. C. Curtis and the alignment group conducted the high precision gyrotheodolite measurements necessary to attain the spin-based energy measurement results. This work was supported by the U.S. DOE Contract No. DE-AC05-84-ER40150.

[1] L. G. Levchuk, Nucl. Instrum. Methods Phys. Res., Sect. A 345, 496 (1994).

[2] M. Swartz et al., Nucl. Instrum. Methods Phys. Res., Sect. A 363, 526 (1995).

[3] C.W. Leemann, D. R. Douglas, and G. A. Krafft, Annu. Rev. Nucl. Part. Sci. 51, 413 (2001).

[4] J. S. Price et al., in Proceedings of the 12th International Symposium on High-Energy Spin Physics (SPIN 96), Amsterdam, Netherlands, 1996 (World Scientific, Singapore, 1996), pp. 727-729.

[5] J. S. Price et al., in Proceedings of the 7th International Workshop on Polarized Gas Targets and Polarized Beams, Urbana, IL, 1997 (AIP, Woodbury, NY, 1997), pp. $446-450$.

[6] M. Steigerwald, in Proceedings of the International Workshop on Polarized Sources and Targets, Erlangen, Germany, 1999 (University of Erlangen-Nurnberg, Erlangen, Germany, 1999), pp. 258-261.

[7] N. Falletto et al., Nucl. Instrum. Methods Phys. Res., Sect. A 459, 412 (2001).

[8] M. Baylac et al., Phys. Lett. B 539, 8 (2002).

[9] HAPPEX, K. A. Aniol et al., Phys. Lett. B 509, 211 (2001).

[10] A.V. Glamazdin, et al., Fizika B 8, 911999.

[11] R. Nasseripour, B. Raue, L. Kramer, and F. Fortier, Bull. Am. Phys. Soc. 45, 91 (2000).

[12] M. Hauger et al., Nucl. Instrum. Methods Phys. Res., Sect. A 462, 382 (2001).

[13] L. J. deBever, M. Loppacher, J. Zhao, W. A. Tobias, and B. Zihlmann, in Proceedings of the 12th International Symposium on High-Energy Spin Physics (SPIN 96), Amsterdam, Netherlands, 1996 (Ref. [4]), pp. 768-770.

[14] C. K. Sinclair, in Proceedings of the 1999 IEEE Particle Accelerator Conference (PAC 99), New York (IEEE, Piscataway, NJ, 1999), pp. 65-69.

[15] M. Poelker et al., in Proceedings of the 14th International Spin Physics Symposium (SPIN 2000), Osaka, Japan, 2000 (AIP, Melville, NY, 2000), pp. 943-948.

[16] M. Salomaa and H. Enge, Nucl. Instrum. Methods 145, 279 (1977). 
[17] K. Halbach, Lawrence Livermore National Laboratory Technical Report No. UCRL-17436, 1967.

[18] J. M. Grames, in Proceedings of the International Workshop on Polarized Sources and Targets, Erlangen, Germany, 1999 (Ref. [6]), pp. 266-269.

[19] J. M. Grames, Ph.D. thesis, University of Illinois at Urbana-Champaign, 2000, ftp://ftp.jlab.org/pub/ grames/thesis.pdf

[20] M. Poelker, Appl. Phys. Lett. 67, 2762 (1995).

[21] J. Schuler et al., in Proceedings of the Low Energy Polarized Electron Workshop (LE98), St. Petersburg, Russia, 1998 (SPES Lab Publishing, St. Petersburg, Russia, 1998).

[22] A. Krycuk, J. Fugitt, A. Johnson, R. Kazimi, and L. Turlington, in Proceedings of the 1993 IEEE Particle Accelerator Conference (PAC 93), Washington, DC (IEEE, Piscataway, NJ, 1993), pp. 939-940.

[23] J. Chuma, Physica Reference Manual v1.3, Vancouver, Canada, triumf computing document tri-cd-93-01 ed. 1998, http://www.triumf.info:8081/physica/html/ homepage.html

[24] W. Press, S. Teukolsky, W. Vetterling, and B. Flannery, Numerical Recipes in Fortran 77 (Cambridge University Press, New York, 1992), 2nd ed.

[25] P. Bevington, Data Reduction and Error Analysis for the Physical Sciences (McGraw-Hill, New York, 1969).

[26] J. Berthot and P. Vernin, Nucl. Phys. News 9N4, 12 (1999).

[27] D. Hardy, J. Tang, L. R. M. Tiefenback, M. Crofford, and G. A. Krafft, in Proceedings of the 1997 IEEE Particle Accelerator Conference (PAC 97), Vancouver, B.C., Canada (IEEE, Piscataway, NJ, 1997), pp. 22652267.

[28] R. Kazimi (private communication).

[29] C. Curtis, Jefferson Laboratory Technical Report, 2000, survey and alignment data transmittals No. L625, No. L634, and No. L643. 